\title{
Computation of Galois groups of rational polynomials
}

\author{
Claus Fieker and Jürgen Klüners
}

\begin{abstract}
Computational Galois theory, in particular the problem of computing the Galois group of a given polynomial, is a very old problem. Currently, the best algorithmic solution is Stauduhar's method. Computationally, one of the key challenges in the application of Stauduhar's method is to find, for a given pair of groups $H<G$, a $G$-relative $H$-invariant, that is a multivariate polynomial $F$ that is $H$-invariant, but not $G$-invariant. While generic, theoretical methods are known to find such $F$, in general they yield impractical answers. We give a general method for computing invariants of large degree which improves on previous known methods, as well as various special invariants that are derived from the structure of the groups. We then apply our new invariants to the task of computing the Galois groups of polynomials over the rational numbers, resulting in the first practical degree independent algorithm.
\end{abstract}

\section{Introduction}

Computational Galois theory, in particular the problem of finding the Galois group of a given polynomial, is a very old problem. While various algorithms have been published, so far they are either impractical for groups of size $>1000$ due to the requirement of exact representation of an algebraic splitting field, or they are degree dependent. Algorithms of the first kind include for example the naive approach of constructing a splitting field by repeated factorization as well as more sophisticated methods [24]. Algorithms of the second kind fall broadly into two approaches: a classical approach that aims to characterize the Galois group as an abstract group by building a decision tree using certain indicators (resolvent polynomials) [4, Chapter 6.3] and a newer approach, by Stauduhar [23] where the Galois group is constructed explicitly as a group of permutations of the roots of the polynomial in question. Stauduhar's method roughly works by traversing the lattice of (transitive) subgroups of the full symmetric group from the top $\left(\mathbf{S}_{n}\right)$ down to the Galois group of the polynomial. At each step, this is done through the help of invariants and the high precision evaluation of those.

This paper naturally splits into two parts: the first discussing the problem of finding a useful invariant for each pair of groups (see $\S 2$ for a precise statement), and the second part explaining how this is used to compute Galois groups of polynomials over $\mathbb{Q}$, see $\S 7$ for details.

Primitive invariants for permutation groups, that is multivariate polynomials with a given stabilizer, are among the most important objects in computational Galois theory. They are the central ingredient in Stauduhar's method $[\mathbf{1 1}, \mathbf{1 2}]$ for the determination of the Galois group of a polynomial $f$ : given two groups $H<G$ a ( $G$-relative) $H$-invariant is used to decide if $\operatorname{Gal}(f) \leqslant H^{g}$ for some $g \in G$ under the assumption that $\operatorname{Gal}(f) \leqslant G$. Furthermore, applications, such as the explicit realization of Galois groups by explicitly computing defining equations for subfields of the splitting field for $f$, rely on invariants as well [18].

While there are a few methods known for the computation of such invariants in the literature, in applications, invariants were mostly the result of ad-hoc methods. Generic algorithms, for example, $[\mathbf{1}, \mathbf{1 4}]$ for individual invariants or $[\mathbf{1 6}]$ for the computation of the entire ring of invariants, become rapidly impractical for larger degree permutation groups.

Received 21 September 2012; revised 19 August 2013.

2010 Mathematics Subject Classification 11R32 (primary), 13B05, 11Y40 (secondary). 
It should be stressed that while invariant theory gives explicit invariants for all pairs of groups $H<G$, the generic results tend to be impractical as the resulting invariants are computationally far too complex.

In what follows, we will give a new, space-efficient algorithm to compute all invariants of a given degree for arbitrary pairs of groups, and for maximal subgroups of transitive groups we give several constructions that allow the determination of efficient invariants in many cases. We then demonstrate in $\S 5$ that knowledge of the subgroup structure can also be used to find efficient invariants, as frequently invariants for some subgroups can be combined to give invariants for others.

Finally, we demonstrate the efficiency and the limits of our methods by considering several examples.

\section{Notation}

Transitive groups of degree $<32$ are denoted by $n T m$ where $n$ is the degree and $m$ is the number of the group in the classification [5] used by both Magma and Gap. For the rest of the article, we fix some positive integer $n$. The symmetric group on $n$ elements, $\mathbf{S}_{n}$, acts on the polynomial ring $\mathbb{Z}[\underline{X}]=\mathbb{Z}\left[X_{1}, \ldots, X_{n}\right]$ in $n$ variables via

$$
X_{i} \mapsto X_{\sigma(i)} .
$$

For $\sigma \in \mathbf{S}_{n}$ we usually write $F^{\sigma}$ for the image under this map. A polynomial $F \in \mathbb{Z}[\underline{X}]$ is called a $H$-invariant (for some group $H \leqslant \mathbf{S}_{n}$ ) if $F^{\sigma}=F$ for all $\sigma \in H$. Given two subgroups $H<G \leqslant \mathbf{S}_{n}$, we call a polynomial $F \in \mathbb{Z}[\underline{X}]$ a $G$-relative $H$-invariant, if its stabilizer $\operatorname{Stab}_{G} F:=$ $\left\{\sigma \in G \mid F^{\sigma}=F\right\}$ in $G$ equals $H$. A polynomial $F \in \mathbb{Z}[\underline{X}]$ is called an absolute $H$-invariant if $\operatorname{Stab}_{\mathbf{s}_{n}} F=H$.

For any subgroup $H \leqslant \mathbf{S}_{n}$ we can consider the ring $\mathbb{Z}[\underline{X}]^{H}$ of absolute $H$-invariants and also the invariant field $\mathbb{Q}(\underline{X})^{H}$ of rational functions that are invariant under $H$.

REMARK 2.1. If $H<G \leqslant \mathbf{S}_{n}$ is a pair of subgroups and if $F \in \mathbb{Z}[\underline{X}]$ is a $G$-relative $H$-invariant, then:

(i) as an extension of fields, $\mathbb{Q}(\underline{X})^{H}$ is a finite extension of $\mathbb{Q}(\underline{X})^{G}$ of degree

$$
\left[\mathbb{Q}(\underline{X})^{H}: \mathbb{Q}(\underline{X})^{G}\right]=(G: H) ;
$$

(ii) furthermore

$$
\mathbb{Q}(\underline{X})^{H}=\mathbb{Q}(\underline{X})^{G}[F]
$$

that is, $F$ is a primitive element for the extension;

(iii) from the main theorem on symmetric functions it follows that

$$
\mathbb{Z}[\underline{X}]^{\mathbf{S}_{n}}=\mathbb{Z}\left[s_{1}, \ldots, s_{n}\right]
$$

where $s_{i}=\sum_{1 \leqslant j_{1}<\ldots<j_{i} \leqslant n} \prod_{\ell=1}^{i} X_{j_{\ell}}$ are the elementary symmetric functions.

\section{Stauduhar's method}

In this section we recall the necessary tools from Stauduhar's method. We do this in a slightly more general context which has the advantage that we can combine the information obtained by the resolvent method and by Stauduhar's method.

Let us assume that we are given a monic polynomial $f \in \mathbb{Z}[X]$ of degree $n$ and we would like to compute the Galois group of $f$. Certainly, the Galois group is a subgroup of $\mathbf{S}_{n}$ acting on 
the roots of $f$ and therefore we can assume that we know a subgroup $G \leqslant \mathbf{S}_{n}$ with $\operatorname{Gal}(f) \leqslant G$. Assume furthermore that we have a proper subgroup $H<G$ and let $F \in \mathbb{Z}\left[X_{1}, \ldots, X_{n}\right]$ be a $G$-relative $H$-invariant polynomial. In the following we denote by $G / / H$ a set of representatives of right cosets $H \sigma$ of $G / H$. We remark that we only use right cosets in this paper. The following is proved in [23].

Lemma 3.1. Let $F$ be $G$-relative $H$-invariant and assume that $\operatorname{Gal}(f) \leqslant G$, where $\operatorname{Gal}(f)$ acts on the roots $\alpha_{1}, \ldots, \alpha_{n}$ in some fixed closure. Then

$$
R_{F}:=\prod_{\sigma \in G / / H}\left(T-F^{\sigma}\left(\alpha_{1}, \ldots, \alpha_{n}\right)\right) \in \mathbb{Z}[T] .
$$

$R_{F}$ is called the relative resolvent polynomial (corresponding to $H<G$ and $F$ ).

Proof. Since $F^{H}=F$ we see that $R_{F}$ does not depend on the choice of coset representatives. The polynomial $R_{F}$ is invariant under $G$ and since $\operatorname{Gal}(f) \leqslant G$ it is invariant under $\operatorname{Gal}(f)$. Therefore all coefficients of $R_{F}$ are in $\mathbb{Q}$ and also algebraic integers, thus in $\mathbb{Z}$.

Suppose that $R_{F}$ is squarefree and we know a non-trivial factor of $R_{F}$ in $\mathbb{Z}[T]$. In this situation we show in the following theorem that the Galois group of $f$ is contained in a proper subgroup of $G$ and therefore we make progress. In case $R_{F}$ is not squarefree, we apply a Tschirnhausen transformation $t \in \mathbb{Z}[x]$ and compute a new polynomial

$$
R_{F, t}:=\prod_{\sigma \in G / / H}\left(T-F^{\sigma}\left(t\left(\alpha_{1}\right), \ldots, t\left(\alpha_{n}\right)\right)\right) .
$$

It can be shown [13] that there exist suitable transformations $t$ such that $R_{F, t}$ is squarefree. Furthermore, introducing $t$ amounts to a change of $f$ that will not affect the Galois group.

Theorem 3.2. In the situation of Lemma 3.1, assume that $R_{F}$ is squarefree and $A \in \mathbb{Z}[T]$ is a divisor of $R_{F}$, of degree $\operatorname{deg} A=m$. Denote by $\rho: G \rightarrow \mathbf{S}_{G / H}$ the permutation action on right cosets $G / H$. Then there exist $\sigma_{1}, \ldots, \sigma_{m} \in G$ such that

$$
A(T)=\prod_{i=1}^{m}\left(T-F^{\sigma_{i}}\left(\alpha_{1}, \ldots, \alpha_{n}\right)\right) .
$$

Denote by $B$ the set of right cosets $\left\{H \sigma_{i} \mid 1 \leqslant i \leqslant m\right\}$.

Then $\operatorname{Gal}(f) \leqslant \rho^{-1}\left(\operatorname{Stab}_{\rho(G)}(B)\right)$.

Proof. The elements $\sigma_{1}, \ldots, \sigma_{m}$ are in pairwise different right cosets of $G / H$ since otherwise $F^{\sigma_{i}}=F^{\sigma_{j}}$ and the polynomial $A$ is not squarefree. Extend the $\sigma_{i}$ to a complete system of representatives $\sigma_{1}, \ldots, \sigma_{r}$ of $G / H$, where $r=(G: H)$. Now let $\tau \in \operatorname{Gal}(f) \leqslant G$ be an arbitrary element. The elements $\tau \sigma_{1}, \ldots, \tau \sigma_{r}$ are also a set of representatives of $G / H$. Since $A$ is invariant under $\tau \in \operatorname{Gal}(f)$, for $1 \leqslant i \leqslant m$ we have $\tau \sigma_{i} \in H \sigma_{j}$ with $j \leqslant m$. Therefore we get that $\rho(\tau) \in$ $\operatorname{Stab}_{\rho(G)}(B)$.

The case of linear factors in Theorem 3.2 was already proved in [23], in fact it formed the key technique in the original paper. The possible use of quadratic factors is mentioned on the last page of [22] but is rejected there since the practical group theory would have been too complicated. The general statement is also proven in [11, Satz 2.4], although only the case of linear factors is used to determine groups. Higher degree factors are only considered in a verification step.

We can also apply this theorem if we know more than one factor. 
Corollary 3.3. Assume that $R_{F}$ is squarefree and factors as $R_{F}=A_{1} \ldots A_{s}$ with $A_{i} \in \mathbb{Z}[T]$. Denote by $B_{i}$ the set of right cosets of $G / H$ corresponding to $A_{i}$. Then

$$
\operatorname{Gal}(f) \leqslant \bigcap_{i=1}^{s} \rho^{-1}\left(\operatorname{Stab}_{\rho(G)} B_{i}\right) .
$$

Because of its importance we describe the case of linear factors in more detail in the following corollary.

Corollary 3.4. Assume that $R_{F}$ is squarefree and has a linear factor in $\mathbb{Z}[T]$ corresponding to $H \sigma$ for $\sigma \in G$. Then $\operatorname{Gal}(f) \leqslant H^{\sigma}:=\sigma^{-1} H \sigma$.

Proof. Note that a point stabilizer of $\rho(G)$ is isomorphic to $H$.

We note that in the following we mostly use this corollary since finding linear factors is much easier than doing a complete factorization. In particular, we are frequently able to find linear factors without ever constructing $R_{F}$ completely. We note that the complexity of this method depends on the index $(G: U)$. Even if we do not compute the corresponding resolvent polynomial of degree $(G: U)$ directly, the coefficient bounds that we need in our algorithm are dependent on this index, too.

If the index $(G: U)$ is huge it could be nice to work with a subgroup $H$ of smaller index and use higher degree factors in order to prove that $\operatorname{Gal}(f)$ is contained in a conjugate of $U$.

EXAMPLE 3.5. Let $f \in \mathbb{Z}[X]$ be a polynomial with Galois group $19 \mathrm{~T} 5 \cong C_{19} \rtimes C_{9}$. This is a maximal subgroup of $\mathbf{A}_{19}$ of index 17 !. Since $19 T 5$ is not 2-transitive, take $S:=\operatorname{Stab}_{\mathbf{A}_{19}}([1,2])$ the intersection of the point stabilizers of 1 and 2 , take $F:=x_{1}-x_{2}$ and compute the resolvent $R_{F}$. This is a polynomial of degree $19 \cdot 18$ which has $\alpha_{i}-\alpha_{j}$ as roots for $1 \leqslant i \neq j \leqslant 19$ (assuming that those roots are different). Furthermore, using resultants, $R_{F}$ can be computed symbolically without explicit knowledge of $S$ or $F$. Factorization of $R_{F}$ finds two factors of degree 171 . When we apply Theorem 3.2 we directly descend to the correct Galois group.

Let $\alpha$ be a root of the polynomial $f$ in the last example. The factorization approach of the last example is equivalent to the fact that $f /(x-\alpha) \in \mathbb{Q}(\alpha)[x]$ factorizes into two degree 9 factors.

Very often the factorization approach is not optimal or even feasible since the degree of the resolvent polynomial is too high for efficient factorization. In some situations our algorithm produces a Galois group (as an actual permutation group on the roots) which is only correct with a very high probability. In this situation we can turn to the factorization method in order to check our result, that is to give a proof that the result is mathematically correct. Since we assume knowledge of the action on the roots, it is not necessary to factor the resolvent polynomial. By analysing the proof of Theorem 3.2 we can write down the factor and check if it is in $\mathbb{Z}[X]$ and whether it divides the resolvent polynomial, see $\S 7.4$ for more details. Similar ideas were used by Casperson and McKay [3] to obtain polynomials with Galois group $M_{11}$.

EXAmple 3.6. Let $p$ be a prime number, $G:=\mathbf{S}_{p+1}$, and $H:=\operatorname{PGL}(2, p) \leqslant G$. Then $(G: H)=(p-2)$ ! and $H$ is a maximal subgroup. Furthermore $G$ is sharply 3 -transitive which means that for the resolvent method we have to use a polynomial acting on 4-sets of the roots which has degree $\left(\begin{array}{c}p+1 \\ 4\end{array}\right)$. For $p=19$ this polynomial has degree 4845 and splits into four factors of degree 570, 855, 1710, and 1710 respectively. In our implementation we compute the Galois group using short cosets (see Remark 7.1) with a very high probability. By applying the methods of $\S 7.4$ we use this group to approximate the degree 570 factor. When computing the corresponding stabilizer according to Theorem 3.2 we descend to $G$. 
We note that the group $\operatorname{PSL}(2, p) \leqslant \mathbf{A}_{p+1}$ is not 3 -transitive. The 3 -set polynomial, that is the resolvent corresponding to $S:=\operatorname{Stab}_{\mathbf{A}_{p+1}}(\{1,2,3\})$, gives no information since this polynomial stays irreducible. But if we take the pointwise stabilizer $S:=\operatorname{Stab}_{\mathbf{A}_{p+1}}[1,2,3]$ (intersection of the three pointwise stabilizers) then we will find three factors. The latter polynomial has only degree $(p+1) p(p-1)$ compared to $\left(\begin{array}{c}p+1 \\ 4\end{array}\right)$.

As a final example in this section we consider a degree 40 polynomial with Galois group PGsp $(4,3)$. This polynomial was computed in [8] and for reasons of space we do not give the actual polynomial here. It comes from the 3 -torsion of a hyperelliptic curve

$$
C: y^{2}+\left(-x^{2}-1\right) y=x^{5}-x^{4}+x^{3}-x^{2} .
$$

The Galois group is primitive and not 2-transitive. Furthermore this group is maximal in $\mathbf{A}_{40}$. The algorithm outlined below, using only linear factors, computes the Galois group within $50 \mathrm{~s}$. However, factoring a suitable resolvent for the stabilizer of 2-sets completes the computation in only $20 \mathrm{~s}$.

\section{Generic invariants}

We fix two groups $H<G \leqslant \mathbf{S}_{n}$ and assume unless explicitly stated otherwise, that $H$ is a maximal subgroup of $G$. The aim of this section is to find a $G$-relative $H$-invariant $F \in \mathbb{Z}[\underline{X}]$ of small degree and a small number of terms. While the first aim can be obtained easily, the second is more difficult, and will be discussed later. To simplify notation we will write $\sum A$ to mean $\sum_{a \in A} a$ for suitable sets $A$, usually orbits.

The first observation is that it is always easy to write down some invariant. Certainly, every $\mathbf{S}_{n}$-relative invariant is $G$-relative as well for $H \leqslant G \leqslant \mathbf{S}_{n}$.

We start with generic absolute invariants.

LEMMA 4.1.

$$
F:=\sum_{\sigma \in H}\left(\prod_{i=1}^{n-1} X_{i}^{i}\right)^{\sigma}
$$

is an $\mathbf{S}_{n}$-relative $H$-invariant.

While Lemma 4.1 proves the existence of $G$-relative $H$-invariants, these are very expensive invariants from the point of view of evaluation. Even assuming that the powers of the evaluation points are stored, the evaluation of each term needs $n-2$ multiplications, so that in total $\# H(n-2)$ multiplications are necessary. In order to improve on this we make use of the following well-known facts $[6]$.

Theorem 4.2. For any polynomial $I \in \mathbb{Z}[\underline{X}]$, and every subgroup $H \leqslant \mathbf{S}_{n}$, we have that $F(\underline{X}):=\sum\left\{I^{h}(\underline{X}) \mid h \in H\right\}=: \sum I^{H}(\underline{X})$ is $H$-invariant.

For every $H$-invariant polynomial $F \in \mathbb{Q}[\underline{X}]$, there exist monomials $m_{i}$ and coefficients $a_{i} \in \mathbb{Q}$ such that

$$
F=\sum_{i=1}^{r} a_{i} \sum m_{i}^{H} .
$$

Thus invariants of the form $\sum m^{H}$ form a vector space basis for the ring of all invariants.

The invariant ring $\mathbb{Q}[\underline{X}]^{H}$ of $H$-invariants is a graded $\mathbb{Q}$-vector space. The dimensions of the summands can be read off from the Hilbert series

$$
f_{H}(t):=\sum_{i=0}^{\infty} t^{i} \operatorname{dim}\left(R_{H}\right)_{i}
$$

where $\left(R_{H}\right)_{i}=\left\{r \in \mathbb{Q}[\underline{X}]^{H} \mid \operatorname{deg} r=i\right\} \cup\{0\}$. 
The Hilbert series can be computed from the knowledge of the set of the conjugacy classes $C$ of $H$,

$$
f_{H}(t)=\frac{1}{\# H} \sum_{c \in C} \frac{\# c}{\prod_{i=1}^{l}\left(1-x^{c_{i}}\right)^{d_{i}}},
$$

where $\left(c_{i}, d_{i}\right)$ is the cycle structure of any representative of the class $c$ of $H$.

To improve on Lemma 4.1 we will try to find a small invariant as a basis element for some $\left(R_{H}\right)_{d}$ for $d$ as small as possible. Unfortunately, there are pairs of groups, $G=\mathbf{S}_{n}, H=\mathbf{A}_{n}$ for example, where the invariant in Lemma 4.1 can be shown to be of minimal degree.

In the remainder of this section we will develop methods to compute a basis for $\left(R_{H}\right)_{d}$ the vector space of $H$-invariant polynomials of degree $d$ and also for the subspace of $G$-relative polynomials. Our strategy will be to first compute a basis for the $\mathbf{S}_{n}$-invariants and then show how to refine this basis. We start with few observations.

REMARK 4.3.

(i) Let $F \in \mathbb{Z}[\underline{X}]$ be a polynomial and $H \leqslant \mathbf{S}_{n}$ be a group. Then

$$
\sum_{\sigma \in H / / \operatorname{Stab}_{H}(F)} F^{\sigma}=\sum F^{H}
$$

and thus is $H$-invariant.

(ii) Let $m=\prod_{i=1}^{n} X_{i}^{a_{i}}$ be a monomial. Then we have

$$
\operatorname{Stab}_{H}(m)=\bigcap_{a \in\left\{a_{i} \mid 1 \leqslant i \leqslant n\right\}} \operatorname{Stab}_{H}\left(\left\{i \mid a_{i}=a\right\}\right),
$$

thus stabilizers of monomials can be computed as intersections of stabilizers of points or sets. Of course, for $H=\mathbf{S}_{n}$ those stabilizers can be made explicit as direct products of suitable $\mathbf{S}_{m}$ for $m<n$.

(iii) Let $\{1, \ldots, n\}=\bigcup_{i=1}^{r} A_{i}$ be a partition. Then

$$
\operatorname{Stab}_{\mathbf{S}_{n}}\left(A_{1}, \ldots, A_{r}\right) \cong \prod_{i=1}^{r} \mathbf{S}_{A_{i}} .
$$

\section{1. $\mathbf{S}_{n}$-invariants}

In this section we will develop methods to compute a basis for the $\mathbf{S}_{n}$-invariants as well as indicate how to improve on the general method if we want to aim for relative invariants only. The algorithm presented here is similar to the ideas presented in $[\mathbf{1}, \mathbf{1 4}]$.

The key idea here is that the orbit sum

$$
\sum_{\sigma \in \mathbf{S}_{n} / / \operatorname{Stab}(f)} f^{\sigma}
$$

does not depend on the representative $f$ of the full orbit $f^{\mathbf{S}_{n}}$ and that the action of the group on some monomial $m$ only depends on the partition of $\{1, \ldots, n\}$ induced by $m=\prod_{i=1}^{n} X_{i}^{a_{i}}$,

$$
\{1, \ldots, n\}=\bigcup_{a \in\left\{a_{i} \mid 1 \leqslant i \leqslant n\right\}}\left\{i \mid a_{i}=a\right\} .
$$

On the other hand, by giving a partition $\underline{A}:=\left\{A_{i} \mid i\right\}$ of $\{1, \ldots, n\}$ and pairwise different integers $a_{i} \geqslant 0$ the orbit of $m(\underline{a}, \underline{A}):=\prod_{i=1}^{s} \prod_{j \in A_{i}} X_{j}^{a_{i}}$ is uniquely defined by $\underline{A}$ already. Thus to solve our problem of finding a basis for $\left(R_{\mathbf{S}_{n}}\right)_{d}$ we simply need to find all partitions and exponents such that $\sum_{i=1}^{s} a_{i} \# A_{i}=d$. We summarize this in an algorithm. 
Algorithm 4.4. Let $d$ be an integer. The algorithm produces a basis for $\left(R_{\mathbf{S}_{n}}\right)_{d}$.

(i) Let $I:=\{\}$.

(ii) Compute the set $P$ of all partitions of $d$ of length at most $n$.

(iii) For $p \in P$ do

(iv) Let $p=\left(p_{1}, \ldots, p_{i}\right)$. Append $I$ by $\prod_{j=1}^{i} X_{j}^{p_{j}}$.

However, since we are eventually only interested in finding minimal degree invariants we introduce more reductions here. The operation of $\mathbf{S}_{n}$ on $m(\underline{a}, \underline{A})$ does only depend on $\underline{A}$, so for a minimal degree invariant we can also stipulate that $a_{i}+1=a_{i+1}$, otherwise the same behaviour can be obtained with smaller exponents. Similarly, minimal examples will be such that $\# A_{1} \geqslant \# A_{2} \geqslant \ldots \geqslant \# A_{s}$. As an example: the orbits of $X_{1} X_{2}^{3}$ and of $X_{1} X_{2}^{2}$ are essentially the same, namely the orbit of $\{\{1\},\{2\}\}$. Thus if we are looking for examples of minimal degree, then $X_{1} X_{2}^{3}$ need not be considered.

\section{2. $H$-invariants}

Let $H$ be a subgroup of $\mathbf{S}_{n}$ and $I$ be a set of monomials generating different $\mathbf{S}_{n}$-invariants via orbit sums. Here we address the problem of refining $I$ to contain a (maximal) set of monomials generating $H$-invariants.

Let $m$ be a monomial and $S:=\operatorname{Stab}_{G}(m)$ its stabilizer in some group $\mathbf{S}_{n} \geqslant G \geqslant H$. We use the following theorem.

Theorem 4.5. Let $G \geqslant H$ be groups, $m$ a monomial and $S=\operatorname{Stab}_{G}(m)$ its stabilizer. Furthermore, let $S \backslash G / H$ be the double cosets of $G$ with respect to $S$ and $H$ and let $\left\{g_{i} \mid 1 \leqslant i \leqslant r\right\}$ be a set of representatives (that is, $G=\bigcup_{i=1}^{r} S g_{i} H$ and $S g_{i} H=S g_{j} H$ if and only if $i=j$ ). Then $\left\{m_{i}^{g} \mid 1 \leqslant i \leqslant r\right\}$ generate linearly independent $H$-invariants.

Proof. The linear independence is a direct consequence from the fact that the double coset decomposition induces a decomposition of $m^{G}$ into pairwise disjoint $H$-orbits.

Thus the computation of $H$-invariants is reduced to the computation of $\mathbf{S}_{n}$-invariants followed by a double coset decomposition. While in general double coset decompositions are hard to compute, it is feasible here. We make use of the ladder-technique of [21]: usually to compute double cosets, one computes a coset decomposition with respect to one group and lets the other group act on them, thus the complexity depends on the size of the index of the larger group in $G$. This procedure is frequently helped by computing a descending chain from $G=: S_{0}>\ldots>S_{j}=S$ down to one smaller group, $S$ for example. The action of $H$ on $S \backslash G$ can then be deducted from the action of $H$ on $S_{i+1} \backslash S_{i}$. Unfortunately, it is hard and frequently impossible to find good subgroup chains, that is chains with small indices. The new idea introduced in [21] is to use a ladder rather than a chain, that is to allow up-ward steps as well as down-ward ones. In order to use this technique we therefore have to construct a suitable ladder. This will be achieved by the following procedure.

Algorithm 4.6. Let $G$ be a permutation group acting on $\Omega$ and $A \subseteq \Omega$ be arbitrary. This algorithm will compute a ladder $G_{i}$ such that $G=G_{0}, G_{r}=\operatorname{Stab}_{G}(A)$ and if $G_{i}<G_{i+1}$ then $\# G_{i+1} / \# G_{i} \leqslant \# \Omega$ and $G_{i}>G_{i+1}$ with $\# G_{i} / \# G_{i+1} \leqslant \# \Omega$ otherwise.

(i) Let $B:=\{\}$, and $i:=1, G_{0}:=G$.

(ii) For $a \in A$ do

(iii) Add $a$ to $B$ and compute $G_{i}:=\operatorname{Stab}_{G_{i-1}}\{a\}$.

(iv) If $B \neq\{a\}$ then $G_{i+1}:=\operatorname{Stab}_{G} B$ and set $i:=i+2$. Otherwise set $i:=i+1$.

Proof. Let $A:=\left\{a_{1}, \ldots, a_{n}\right\}$. The properties of the $G_{i}$ are direct consequences of the following facts: 
(i) we either have $G_{i}=\operatorname{Stab}_{G_{i-1}}\left\{\left\{a_{1}, \ldots, a_{s}\right\},\left\{a_{s+1}\right\}\right\}$ (in which case $G_{i}<G_{i+1}$ ); or

(ii) we have an up-ward step and obtain $G_{i+1}=\operatorname{Stab}_{G}\left\{a_{1}, \ldots, a_{s+1}\right\}$. Note that $\operatorname{Stab}_{G_{i+1}}$ $\left\{a_{s+1}\right\}=G_{i}$

(iii) for $G=\mathbf{S}_{n}$, we have \# $\operatorname{Stab}_{G}\left\{a_{1}, \ldots, a_{s}\right\}=s !(n-s)$ ! and \# $\operatorname{Stab}_{G}\left\{\left\{a_{1}, \ldots, a_{s}\right\}\right.$, $\left.\left\{a_{s+1}\right\}\right\}=s ! 1 !(n-s-1) !$

(iv) in general, $\operatorname{Stab}_{G} A=G \cap \operatorname{Stab}_{\mathbf{S}_{n}} A$, and for any groups $V<U<\mathbf{S}_{n}$ we have $(U \cap G$ : $V \cap G) \leqslant(U: V)$, thus the bound on the indices follows.

For more general partitions, Algorithm 4.6 will be called repeatedly.

Algorithm 4.7. Let $G$ be a permutation group acting on $\Omega$ and $A=\left\{A_{1}, \ldots, A_{s}\right\}$ a partition of $\Omega$. This algorithm will compute a ladder $G_{i}$ such that $G=G_{0}, G_{r}=\operatorname{Stab}_{G}(A)$ and if $G_{i}<G_{i+1}$ then $\# G_{i+1} / \# G_{i} \leqslant \# \Omega$ and $G_{i}>G_{i+1}$ with $\# G_{i} / \# G_{i+1} \leqslant \# \Omega$ otherwise.

(i) Let $U:=G$.

(ii) For $a \in A$ do

(iii) Compute a ladder from $U$ to $\operatorname{Stab}_{U} a$ using Algorithm 4.6 and print it.

(iv) Let $U:=\operatorname{Stab}_{U} a$.

Let $G \leqslant \mathbf{S}_{n}$ be arbitrary and $H<G$ a maximal subgroup. In order to compute $G$-relative $H$-invariants, we now use one of the following algorithms.

Algorithm 4.8. Let $H<G$ be as above and $d>0$ be an integer. This algorithm will find a basis for the space of $G$-relative $H$-invariants of degree $d$.

(i) Compute a basis $B$ for $\left(R_{\mathbf{S}_{n}}\right)_{d}$ using Algorithm 4.4.

(ii) For each $b \in B$ do

(iii) Compute the corresponding partition $A$.

(iv) Use Algorithm 4.7 to compute a ladder $L$ from $\mathbf{S}_{n}$ to $\operatorname{Stab}_{\mathbf{S}_{n}}(b)$ using the partition $A$.

(v) Use $L$ to compute a set $C$ of double coset representatives for $\operatorname{Stab}_{\mathbf{S}_{n}} b \backslash \mathbf{S}_{n} / H$.

(vi) For each $c \in C$ do

(vii) Compute the indices of the stabilizers $\left(H: \operatorname{Stab}_{H} b^{c}\right)$ and $\left(G: \operatorname{Stab}_{G} b^{c}\right)$. If they differ then $b^{c}$ generates a $G$-relative $H$-invariant. In this case print $\sum_{h \in H / / \operatorname{Stab}_{H} b^{c}} b^{c h}$.

The correctness of the algorithm follows immediately from the above discussions. We remark that if we want only one invariant rather than a basis, we can use a probabilistic approach.

Algorithm 4.9. Let $H<G$ be as above and $d>0$ be an integer such that there exists an $G$-relative $H$-invariant of degree $d$. This algorithm will find one $G$-relative $H$-invariant of degree $d$.

(i) Compute a basis $B$ for $\left(R_{\mathbf{S}_{n}}\right)_{d}$ using Algorithm 4.4.

(ii) Repeat.

(iii) Compute a random element $\sigma \in \mathbf{S}_{n}$

(iv) For each $b \in B$ check if $\left(G: \operatorname{Stab}_{G} b^{\sigma}\right)$ differs from $\left(H: \operatorname{Stab}_{H} b^{\sigma}\right)$. If so, print $\sum_{h \in H / / \operatorname{Stab}_{H} b^{\sigma}} b^{\sigma h}$ and terminate.

To find a (minimal) degree $d$ such that there exists an $G$-relative $H$-invariant we simply compute the difference of the Molien series $f_{H}(t)-f_{G}(t)=\sum_{i=1}^{\infty} s_{i} t_{i}$ and take $d$ as the index of any non-zero coefficient.

\section{Special invariants}

Like in the previous section we assume that $H$ is a maximal subgroup of $G$. We use the maximality in our proofs to show that an $H$-invariant $F$ is $G$-relative, if there exists one element $g \in G \backslash H$ with $F^{g} \neq F$. 
Unfortunately, there are examples where the generic invariants are too expensive to compute or the given presentation needs too many arithmetic operations to evaluate the invariant. The best known example for this are the groups $H=\mathbf{A}_{n}$ and $G=\mathbf{S}_{n}$. Clearly, $H$ is a maximal subgroup of $G$ and the invariant

$$
F_{1}\left(X_{1}, \ldots, X_{n}\right):=\sum_{\sigma \in H}\left(\prod_{i=1}^{n-1} X_{i}^{i}\right)^{\sigma}
$$

given in Lemma 4.1 is an $\mathbf{S}_{n}$-relative $\mathbf{A}_{n}$-invariant polynomial of smallest possible total degree. If we store the powers of $X_{i}$ we need $(n-2) n ! / 2$ multiplications in order to evaluate this invariant. If the characteristic is not equal to 2 , then a better invariant is well known: (for any $\left.\sigma \notin H=\mathbf{A}_{n}\right)$

$$
F_{2}\left(X_{1}, \ldots, X_{n}\right):=\prod_{1 \leqslant i<j \leqslant n}\left(X_{i}-X_{j}\right)=F_{1}-F_{1}^{\sigma},
$$

which can be evaluated using $n(n-1) / 2$ multiplications, if the factored form is used.

Most of the special invariants presented here follow the same pattern and are derived from the same source, namely from the different action of $G$ and $H$ on natural objects like the action on blocks or block systems. Ultimately, as we saw above in the discussion of general factorization patterns, we can use permutation presentations for $G$ acting on the cosets by any subgroup $V<G$.

In the following we assume that $H<G \leqslant \mathbf{S}_{n}$ where $H$ is maximal in $G$ are acting on $\Omega:=$ $\left\{X_{1}, \ldots, X_{n}\right\}$. Let us start with the case that $H$ is acting intransitively. The proof of the following lemma is trivial.

Lemma 5.1. Assume that there exists an orbit $\mathfrak{O}$ of $H$ on $\Omega$ which is not invariant under $G$. Then

$$
F\left(X_{1}, \ldots, X_{n}\right):=\sum_{X_{i} \in \mathcal{O}} X_{i}
$$

is a $G$-relative $H$-invariant.

We remark that intransitive groups may occur in our applications even if we start with transitive groups. The reason is that some of the following algorithms will reduce the problem recursively to groups of smaller degree.

Let us assume for the rest of the section that the given groups $H \leqslant G \leqslant \mathbf{S}_{n}$ are transitive. For transitive groups the notion of blocks and block systems are very important. We remark that most of the following invariants are well known, for example see $[\mathbf{1 1}, \mathbf{1 2}]$.

Definition 5.2. Let $G \leqslant \mathbf{S}_{n}$ be transitive and $\emptyset \neq B \subseteq \Omega$ be a subset. Then $B$ is called a block, if for all $g \in G$ we have $B^{g} \cap B:=\left\{X^{g} \mid X \in B\right\} \cap B \in\{\emptyset, B\}$. Blocks of size 1 and $n$ are called trivial blocks.

It is very easy to see that $B^{g}$ is a block if $B$ is a block. By acting on a block $B$ we get a partition of $\Omega$ which is called a block system. Therefore every block is contained in a block system. Furthermore it is easy to see that the blocks containing $X_{1}$ are in 1-1 correspondence to the groups $G_{X_{1}} \leqslant U \leqslant G$, where $G_{X_{1}}=\operatorname{Stab}_{G}\left\{X_{1}\right\}$ is the point stabilizer of $G$ and $U$ is the stabilizer of the block, that is $U=\operatorname{Stab}_{G} B=\left\{g \in G \mid B^{g}=B\right\}$.

If $H \leqslant G$ then clearly every block (system) of $G$ is a block (system) of $H$. But it may be the case that $H$ possesses more blocks. 
Lemma 5.3. Let $H \leqslant G \leqslant \mathbf{S}_{n}$ be transitive groups and assume that $B_{1}, \ldots, B_{m}$ is a block system of $H$, but not one of $G$. Then

$$
F\left(X_{1}, \ldots, X_{n}\right):=\prod_{i=1}^{m} \sum_{X \in B_{i}} X
$$

is a $G$-relative $H$-invariant.

Proof. Every $h \in H$ only permutes the factors of $F$ and therefore stabilizes $F$. Let $g \in G \backslash H$. Then there exist $X_{i}$ and $X_{j}$ lying in the same block which are mapped to different blocks. This produces a monomial of $F^{g}$ containing $X_{i} X_{j}$ which does not exist in $F$. Since cancellations are impossible, we get the desired result.

Now we can assume that the block systems of $H$ and $G$ coincide. Now let $B_{1}, \ldots, B_{m}$ be a block system of $H$ (and $G$ ). We can define two canonical actions of $G$ and $H$. One is by simply permuting the blocks which give transitive permutation representations $\bar{G}$ and $\bar{H}$ on $m$ points. We get the following exact sequences of groups,

$$
1 \rightarrow N_{G} \rightarrow G \rightarrow \bar{G} \rightarrow 1, \quad 1 \rightarrow N_{H} \rightarrow H \rightarrow \bar{H} \rightarrow 1,
$$

where $N_{G}$ (respectively $N_{H}$ ) is the kernel of the permutation representation.

In the case that $N_{H}=N_{G}$ we can apply the following lemma. We remark that we always get $N_{H}=N_{G}$ if $\bar{H} \neq \bar{G}$. This is true because $H$ is a maximal subgroup of $G$ by our general assumption.

Lemma 5.4. Let $H \leqslant G \leqslant \mathbf{S}_{n}$ be transitive groups with a common block system $B_{1}, \ldots, B_{m}$. Assume that the above defined normal subgroups $N_{H}$ and $N_{G}$ are equal. Let $E\left(X_{1}, \ldots, X_{m}\right)$ be a $\bar{G}$-relative $\bar{H}$-invariant. Then

$$
F\left(X_{1}, \ldots, X_{n}\right):=E\left(Y_{1}, \ldots, Y_{m}\right) \text { for } Y_{i}:=\sum_{X \in B_{i}} X
$$

is a $G$-relative $H$-invariant.

Proof. Elements of $N_{H}=N_{G}$ only change the ordering of the sum defining $Y_{i}$. Therefore an element $g$ acts on $F$ via the action of $\bar{g}$ on $E$. Therefore the polynomial $F$ is $H$-invariant. In order to show the $G$-relativity, we need to prove that for $g \in G \backslash H$ we have $\bar{g} \notin \bar{H}$. The last statement easily follows from $N_{H}=N_{G}$.

The other action can be defined within a block $B_{1}$ via $\left.\operatorname{Stab}_{G}\left(B_{1}\right)\right|_{B_{1}}$. We get the following invariant.

Lemma 5.5. Let $H \leqslant G \leqslant \mathbf{S}_{n}$ be transitive groups with a common block system $B_{1}, \ldots, B_{m}$. Let $\tilde{H}:=\left.\operatorname{Stab}_{H}\left(B_{1}\right)\right|_{B_{1}}, \tilde{G}:=\left.\operatorname{Stab}_{G}\left(B_{1}\right)\right|_{B_{1}}$ and assume $[G: H]=[\tilde{G}: \tilde{H}]$. Let $E\left(X_{i_{1}}, \ldots, X_{i_{l}}\right)$ where $B_{1}=\left\{X_{i_{1}}, \ldots, X_{i_{l}}\right\}$ is a $\tilde{G}$-relative $\tilde{H}$-invariant. Furthermore let $\left\{\sigma_{1}, \ldots, \sigma_{m}\right\}$ be a system of representatives of right cosets of $\operatorname{Stab}_{H}\left(B_{1}\right)$ in $H$.

Then $F:=E^{\sigma_{1}}+\ldots+E^{\sigma_{m}}$ is a $G$-relative $H$-invariant.

Proof. An element of $H$ can be uniquely written as a product of an element of $\operatorname{Stab}_{H}\left(B_{1}\right)$ and some $\sigma_{i}$. The first one stabilizes $E$ and the second one only permutes the $E^{\sigma_{i}}$. Therefore $F$ is invariant under $H$. Since $[G: H]=[\tilde{G}: \tilde{H}]$ we see that $\left\{\sigma_{1}, \ldots, \sigma_{m}\right\}$ are representatives of the right cosets of $\operatorname{Stab}_{G}\left(B_{1}\right)$ in $G$. Since an element $g \in G \backslash H$ can be uniquely written as a product $\tilde{g} \sigma_{i}$ of an element $\tilde{g} \in \operatorname{Stab}_{G}\left(B_{1}\right)$ and some $\sigma_{i}$ we get that the element $\tilde{g}$ cannot be 
an element of $\operatorname{Stab}_{H}\left(B_{1}\right)$. Therefore $E^{\tilde{g}} \neq E$. Furthermore the $X_{j}$ which appear in $E^{\sigma_{i}}$ are different for different values of $i$ which shows that $F^{g} \neq F$.

Now we have to deal with groups where the number of block systems is the same and it is not possible to use Lemmas 5.4 or 5.5. In this situation, we can try the following $[\mathbf{1 1}, 6.19]$ in the situation that the size of $O$ is not too large.

Lemma 5.6. Let $U:=\left.\operatorname{Stab}_{H}\left(B_{1}\right)\right|_{B_{1}}=\left.\operatorname{Stab}_{G}\left(B_{1}\right)\right|_{B_{1}}$ and $K_{1}<K_{2} \leqslant U$. Now let $F$ be a $K_{2}$-relative $K_{1}$-invariant such that $O:=F^{G}=F^{H}$ and the orbit $O$ has the form $\left\{F^{\sigma_{1}}, \ldots, F^{\sigma_{o}}\right\}$ for suitable elements $\sigma_{1}, \ldots, \sigma_{o} \in H$. Finally let $\rho: G \rightarrow \mathbf{S}_{O}$ be the permutation representation of $G$ on $O$. If $\rho(H) \neq \rho(G)$ then let $Y$ be a $\rho(G)$-relative $\rho(H)$-invariant. For a suitable Tschirnhausen transformation $t \in \mathbb{Z}[x]$ we have that

$$
I:=Y\left(t\left(F^{\sigma_{1}}(\underline{X})\right), \ldots, t\left(F^{\sigma_{o}}(\underline{X})\right)\right)
$$

is a $G$-relative $H$-invariant.

Proof. Since $G$ and $H$ act identically on the block $B_{1}$, the orbits $F^{G}$ and $F^{H}$ are the same. By construction, $I$ is clearly $H$-invariant, all that we need to show is that $I$ is not $G$-invariant. Since $F$ is not $\rho(G)$ invariant, this is immediate.

It should be noted that the use of blocks above is only part of the attempt to create an invariant $F$ with a small orbit.

The following theorem is a generalization of a result of Eichenlaub [9], who proved the corresponding result for wreath products of symmetric groups. Recall that a wreath product $U \smile V$ is a semidirect product of the type $U^{m} \rtimes V$, where $V \leqslant S_{m}$ and the action of $V$ permutes the copies of $U$. For a formal definition we refer the reader to [7, p. 46].

Theorem 5.7. Let $G=U<V$ be the wreath product acting on $X_{i, j}(1 \leqslant i \leqslant d, 1 \leqslant j \leqslant m)$, where $U \leqslant S_{d}, V \leqslant S_{m}$ and $m d=n$. Furthermore let $N \unlhd U$ be a normal subgroup of index 2 . Let $E$ be a $U$-relative $N$-invariant with the property that $E^{u}=-E$ for all $u \in U \backslash N$. Denote by $s_{k}$ the $k$ th elementary symmetric function on $m$ letters. Then $G$ has a subgroup $H$ of index 2 and

$$
F\left(X_{1,1}, \ldots, X_{d, m}\right):=s_{m}\left(d_{1}, \ldots, d_{m}\right)=d_{1} \ldots d_{m}
$$

is a $G$-relative $H$-invariant, where $d_{j}:=E\left(X_{1, j}, \ldots, X_{d, j}\right)$.

We note that in the original statement given in [9] there are two other subgroups of index 2. One is $S_{d} \prec A_{m} \leqslant S_{d} 2 S_{m}$ which can be dealt with by Lemma 5.4 and the other one comes from the fact that whenever we have two subgroups of index 2 , there will be a third one. An invariant for this can be efficiently computed using the first two invariants, see Lemma 5.8.

Proof. Clearly, we have $N \imath V \leqslant U \imath V$ and using Lemma 5.5 we get that $E+E^{u}$ with $u \in U \backslash N$ is a $G$-relative $N \imath V$-invariant. Let $u \in U \backslash N$ be an arbitrary element and let $u_{1}$ and $u_{2}$ be the canonical images of $u$ in the first and second copy of $U^{m}$ in $G$, respectively. Now we claim that $H=\left\langle N \backslash V, u_{1} u_{2}\right\rangle \leqslant G$. Clearly, $F$ fixes all elements of $N \imath V$ because all $d_{i}$ are fixed by elements of $N$ and swapped by elements of $V$. The element $u_{1} u_{2}$ fixes $d_{3}, \ldots, d_{m}$ and has the property that $d_{1}^{u_{1} u_{2}}=-d_{1}$ and $d_{2}^{u_{1} u_{2}}=-d_{2}$. Therefore we get $F^{u_{1} u_{2}}=F$. For an arbitrary element $g \in G$ we get that $F^{g}= \pm F$ and therefore the index of $H$ in $G$ is at most 2. Clearly, $F^{u_{1}}=-F$ and therefore $H \neq G$ and $F$ is $G$-relative.

We note that this invariant can be applied to groups $G$ which are not wreath products. For example, it could be possible that $G$ is contained in a wreath product, but not in the index 2 -subgroup and $H$ is contained in that index 2-subgroup. 
As already mentioned it is possible to combine relative invariants in order to get new ones. Suppose $G \leqslant \mathbf{S}_{n}$ has two subgroups $H_{1}<G$ and $H_{2}<G$ with $G$-relative $H_{i}$-invariants $F_{i}$. On the invariant field side, this corresponds to $\mathbb{Q}(\underline{X})^{G}$ having two finite separable extensions $\mathbb{Q}(\underline{X})^{G}\left(F_{i}\right)$ corresponding to $\mathbb{Q}(\underline{X})^{H_{i}}$ with normal closures $M^{C_{i}}$ and $C_{i}:=$ Core $_{G}\left(H_{i}\right)$. In this situation we can transfer information about $H_{i}$ to all subfields (and the corresponding fix groups) of the compositum $M^{C_{1}} M^{C_{2}}=M^{\text {Core }_{G}\left(H_{1} \cap H_{2}\right)}$.

The first such example already appears in $[\mathbf{9}]$.

Lemma 5.8. Let $G \leqslant \mathbf{S}_{n}$ be a permutation group which has two subgroups $H_{1} \neq H_{2}$ of index 2 with $G$-relative $H_{i}$-invariants $F_{i}$. If $F_{i}^{g}= \pm F_{i}$ for $g \in G$, then $F_{1} F_{2}$ is a $G$-relative $H_{3}$-invariant for $H_{3}:=\left(H_{1} \cap H_{2}\right) \cup\left(\left(G \backslash H_{1}\right) \cap\left(G \backslash H_{2}\right)\right)$.

Proof. An element of $H_{1} \cap H_{2}$ clearly stabilizes $F$. Therefore let $h \in H_{3} \backslash H_{1} \cap H_{2}$. Then $h \notin H_{1} \cup H_{2}$ and therefore $F_{1}^{h}=-F_{1}$ and $F_{2}^{h}=-F_{2}$ which gives $F^{h}=F$. This proves that $F$ is $H_{3}$-invariant. Let $g \in G \backslash H_{3}$. Then $g \in H_{1}$ or $g \in H_{2}$, but $g \notin H_{1} \cap H_{2}$. Therefore $F^{g}=-F$ and $F$ is $G$-relative.

Even if the invariants do not satisfy $F_{i}^{g}= \pm F_{i}$, the above Lemma 5.8 can be used, since for $G / / H_{i}=\{\mathrm{Id}, g\}$ we see that $\tilde{F}_{i}:=F_{i}-F_{i}^{g}$ is a $G$-relative $H_{i}$-invariant with the desired property $\tilde{F}_{i}^{g}=-\tilde{F}_{i}$.

In the more general situation we can still use the field theoretic view to combine information from two (or more) subgroups. Assume $G<\mathbf{S}_{n}$ has two subgroups $H_{i}<G(i=1,2)$ with $G$-relative $H_{i}$-invariants $F_{i}$, set $H_{12}:=H_{1} \cap H_{2}$ and $C_{i}:=\operatorname{Core}_{G}\left(H_{i}\right)$ for $i \in\{1,2,12\}$. Then for any maximal subgroup $C_{12}<H_{3}<G$ a $G$-relative $H_{3}$ invariant can be constructed by any of the following methods from $F_{i},(i=1,2)$ and a $G / C_{12}$-relative $H_{3} / C_{12}$-invariant. Also, set $K:=\mathbb{Q}(\underline{X})^{G}$.

(1) Intransitive construction. Let $\tilde{H}_{i}$ be the permutation representation of $G$ on $G / / H_{i}$, $i \in\{1,2,12\}$. We consider the subdirect product $\tilde{H}_{1} \times_{H_{12}} \tilde{H}_{2} \cong \tilde{H}_{12} \cong G / C_{12}$. The maximal subgroup $H_{3}<G$ corresponds to a maximal subgroup $\tilde{H}_{3}<\tilde{H}_{12}$. Let $F$ be an invariant for this pair, then $F\left(\left[F_{1}^{s}: s \in G / / H_{1}\right],\left[F_{2}^{s}: s \in G / / H_{2}\right]\right)$ is a $G$-relative $H_{3}$-invariant.

(2) Transitive construction. By the primitive element theorem, we can find an invariant $F_{i}$, $i=1,2$, such that $K\left(F_{i}\right)=\mathbb{Q}(\underline{X})^{C_{i}}$. Again, by the primitive element theorem, we find some $r$ such that $F_{1}+r F_{2}$ is primitive for $\mathbb{Q}(\underline{X})^{C_{12}}$. From here it is straight forward to obtain an invariant for $H_{3}$ as a polynomial in $F_{1}+r F_{2}$.

In general, this is only applicable if the indices of the groups in question are small. In particular, the transitive construction is mainly of interest for normal subgroups.

\section{Intransitive groups}

The Stauduhar algorithm works for intransitive groups (from reducible polynomials) in the same way as it does for transitive groups (from irreducible polynomials). Let $f \in \mathbb{Q}[x]$ be a squarefree polynomial of degree $n$. Assume that $f=f_{1} \ldots f_{r} \in \mathbb{Q}[x]$ has $r$ factors of degree $n_{i}=\operatorname{deg} f_{i}$. Then we know that $\operatorname{Gal}(f)$ is a subgroup of the intransitive group $\mathbf{S}_{n_{1}} \times \ldots \times$ $\mathbf{S}_{n_{r}} \leqslant \mathbf{S}_{n}$. Using the methods for irreducible polynomials we can compute the Galois groups $G_{i}:=\operatorname{Gal}\left(f_{i}\right) \leqslant \mathbf{S}_{n_{i}}$. Then $\operatorname{Gal}(f) \leqslant G_{1} \times \ldots \times G_{r} \leqslant \mathbf{S}_{n}$. This direct product can be used as a starting group of our algorithm.

In order to simplify the presentation, let us assume that $\operatorname{Gal}(f) \leqslant G_{1} \times G_{2}<\mathbf{S}_{n}$. This is no restriction, since we do not assume that $G_{1}$ or $G_{2}$ are transitive. Therefore we have a corresponding factorization $f=f_{1} f_{2} \in \mathbb{Q}[x]$, where we do not assume that $f_{1}, f_{2}$ are irreducible. All groups $H$ with $\operatorname{Gal}(f) \leqslant H \leqslant G_{1} \times G_{2}$ have a special structure. Let us start to theoretically 
describe $\operatorname{Gal}(f)$. Denote by $N_{i}$ the splitting field of $f_{i}$. Furthermore define $N$ to be the compositum $N_{1} N_{2}$ and $M:=N_{1} \cap N_{2}$. Let $U$ be the Galois group of $M / K$. Then the Galois group of $N / M$ is the subdirect product (fibre product) $G_{1} \times_{U} G_{2}$ with common factor group $U$. Denote by $\phi_{i}: G_{i} \rightarrow U$ the corresponding epimorphisms. Then $G_{1} \times_{U} G_{2}$ can be realized via

$$
G_{1} \times_{U} G_{2}=\left\{\left(g_{1}, g_{2}\right) \in G_{1} \times G_{2} \mid \phi_{1}\left(g_{1}\right)=\phi_{2}\left(g_{2}\right)\right\} .
$$

Now let us consider the case that $H=G_{1} \times{ }_{U} G_{2}$ and $G=G_{1} \times G_{2}$. We note [2, Corollary 1.3] that $H$ is a normal subgroup of $G$, if and only if $U$ is abelian. Define $V_{i} \leqslant G_{i}$ to be the normal subgroups such that $G_{i} / V_{i}=U$. Then we get the following chain of subgroups,

$$
V_{1} \times V_{2} \leqslant G_{1} \times_{U} G_{2} \leqslant G_{1} \times G_{2} .
$$

A $G_{1} \times G_{2}$-relative $V_{1} \times V_{2}$-invariant can be computed by using the corresponding $G_{i}$-relative $V_{i}$-invariants defined on the components and the primitive field argument. This invariant can be improved to a $G$-relative $H$-invariant by taking sums over elements from $H / /\left(V_{1} \times V_{2}\right)$.

In general, since the generic invariants are computationally bad, we would like to use special invariants in this case as well. However, none of them work for intransitive groups, so our only chance here is to compute a transitive representation of the larger group and then test for special invariants in the transitive representation. Let $H<G$ and $G$ be intransitive. If the $G$-orbits and the $H$-orbits differ, we get a trivial $G$-relative $H$-invariant from any $H$-orbit that is no $G$-orbit. Hence, we assume that the orbits are the same. Similarly, we assume that the action of $G$ and $H$ on the orbits agree. In this case we construct a transitive representation $\phi: G \rightarrow \mathbf{S}_{T}$ of $G$ on the set $T:=\prod_{o \in O} o$ where the product runs over all orbits. The image $\phi(H)$ of $H$ under this representation is again a subgroup of $\phi(G)$ and we can now test for special invariants.

Lemma 6.1. Assume $I \in \mathbb{Z}\left[X_{t} \mid t \in T\right]$ is a $\phi(G)$-relative $\phi(H)$-invariant. Then there exist a suitable Tschirnhausen transformation $y \in \mathbb{Z}[x]$ such that

$$
I\left(y\left(\sum_{o \in O} X_{t_{o}}\right) \mid t \in T\right)
$$

is a $G$-relative $H$-invariant.

Proof. For any fixed $t \in T$ it is clear that $\sum_{o \in O} X_{t_{o}}$ is a primitive element for $\mathbb{Q}\left[X_{t} \mid t \in\right.$ $T]^{\phi(H)} / \mathbb{Q}\left[X_{t} \mid t \in T\right]^{\phi(G)}$ since all the conjugates $\sum_{o \in O} X_{s_{o}}, s \in T$, are different.

\section{Computation of Galois groups}

In the previous sections we investigated a variety of special and generic constructions for invariants. Here we are going to discuss how they can be used to compute Galois groups.

To start, let $f$ be an irreducible monic polynomial in $\mathbb{Z}[x]$ and let $K$ be an extension of $\mathbb{Q}$ such that $f(x)=\prod_{i=1}^{n}\left(x-\alpha_{i}\right)$ with $\alpha_{i} \in K$, that is $K$ a fixed splitting field, not necessarily a minimal one. We want to compute

$$
\operatorname{Gal}(f):=\operatorname{Aut}\left(\mathbb{Q}\left(\alpha_{1}, \ldots, \alpha_{n}\right) / \mathbb{Q}\right) \leqslant \mathbf{S}_{\alpha_{1}, \ldots, \alpha_{n}} .
$$

Since we assume $f$ to be irreducible, $\operatorname{Gal}(f)$ is a transitive subgroup of $\mathbf{S}_{n}$.

Suitable choices for $K$ are $p$-adic fields or the field of complex numbers. We will defer the choice of the field until we discussed the operations we need to perform with it. Thus we assume that (somehow) we are given a field $K$ and the roots $\alpha_{i}$ in some arbitrary but fixed ordering.

The main algorithm will, starting with some group $G \geqslant \operatorname{Gal}(f)$, refine the initial guess by considering (maximal) subgroups. Hence the first step is a good starting group. 


\subsection{Starting group}

Naively, obviously, $\operatorname{Gal}(f) \leqslant \mathbf{S}_{n}$, so $G:=\mathbf{S}_{n}$ is a valid start. However, this is very bad for the subsequent steps as $\mathbf{S}_{n}$ has maximal subgroups of very large index.

Set $E:=\mathbb{Q}\left(\alpha_{1}\right)=\mathbb{Q}(x) / f$ which is a number field of degree $n$. Using algorithms developed by Klüners [17] (or recently van Hoeij, Klüners and Novocin [15]) it is relatively easy to find subfields $\mathbb{Q} \subset F \subset E$, or to decide that there are no subfields. By Galois theory, the subfields are in 1-1 correspondence to the (unknown) block systems of the (unknown) Galois group. Thus the non-existence of subfields proves the group to be primitive.

Assume we have a non-trivial subfield $F=\mathbb{Q}(\beta)$ for $\beta=\sum r_{\ell} \alpha_{1}^{\ell}$. Then $B_{1}:=\left\{\alpha_{j} \mid \sum r_{\ell} \alpha_{i}^{\ell}=\right.$ $\left.\sum r_{\ell} \alpha_{1}^{\ell}\right\}$ is the block containing $\alpha_{1}$, the other blocks are computed similarly. From here, it is trivial to compute the wreath product $W_{F}$ corresponding to this block system, and $\operatorname{Gal}(f) \subseteq W_{F}$. The construction can be improved if we compute the Galois group of $F / \mathbb{Q}$ (and even more if we compute the group of $F / E$, but this is too expensive in practise). Doing this for all subfields, we compute a suitable starting group.

If there are no subfields, hence $\operatorname{Gal}(f)$ is primitive, we can try to obtain a good starting group from factoring suitable resolvent polynomials as indicated in Theorem 3.2 and Examples 3.5 and 3.6.

\subsection{Stauduhar}

We assume now that we have some $\operatorname{Gal}(f) \leqslant G \leqslant \mathbf{S}_{n}$. The next step is now to either prove that $G$ is the Galois group or replace it by some smaller group $H$. Now let $H<G$ be maximal. If we have subfields, we should also verify that $H$ admits the same block systems as $G$, otherwise it cannot contain the Galois group. In the following we would like to apply Corollary 3.4.

We now find a $G$-relative $H$-invariant $F$ using any of the methods above, typically starting with the special invariants in $\S 5$ and, failing that, using $\S 4$. Next, we verify that

$$
F^{\sigma}(\underline{\alpha})=F^{\tau}(\underline{\alpha}) \quad \text { if and only if } \quad \sigma \tau^{-1} \in H
$$

and compute

$$
C:=\left\{\sigma \in G / / H \mid F^{\sigma}(\underline{\alpha}) \in \mathbb{Z}\right\} .
$$

If $C$ is non-empty, then $G:=\bigcap_{\sigma \in C} H^{\sigma}$ will be our new group.

REMARK 7.1. Obviously, if $(G: H)$ is large, this is going to be very inefficient, if not impossible. If we have knowledge of some non-trivial element $\tau \in \operatorname{Gal}(f)$, coming from some known automorphism of $K$, then we can aid the computation of $C$. Instead of $G / / H$ we only compute

$$
G / /{ }_{\tau} H:=\left\{\sigma \in G / / H \mid \tau \in H^{\sigma}\right\}
$$

the so called short-coset. The actual computation of $G / /{ }_{\tau} H$ can be performed even if $(G: H)$ is too large to be computed $[\mathbf{1 2}, 4.6]$.

However, we do not know how to test (7.1) effectively. All we do here is to apply some probabilistic test, that is test for some 100 cosets if the images differ and rely on an independent proof later to justify the result.

\subsection{Splitting field}

Now that we have looked at the components of the algorithm, we can discuss the splitting field. As we saw, we need to be able to quickly evaluate $F(\underline{\alpha})$, decide if two such evaluations are different and, finally, test if $F(\underline{\alpha}) \in \mathbb{Z}$. All of those tasks would be trivial if we could use a purely algebraic, exact representation of a splitting field $K$. However, since $[K: \mathbb{Q}] \geqslant \# \operatorname{Gal}(f)$ 
this is in general not practical. Using $K=\mathbb{C}$ as Stauduhar did is possible, but makes it difficult to decide if $F(\underline{\alpha}) \in \mathbb{Z}$; this would involve a careful analysis of the numerical properties of $F$. By restricting the invariants to be free of division, and using a suitable $p$-adic field $K$, we can overcome most problems, although we actually need both complex and $p$-adic information. We choose a suitable prime $p$ and compute a finite extension $K$ of $\mathbb{Q}_{p}$. The complex information is used to derive the $p$-adic precision necessary to guarantee correctness. Let $0<M$ be such that $\left|\alpha_{i}\right| \leqslant M$ for all complex roots $\alpha_{i}$. It is now easy to compute $N$ such that $\left|F^{\sigma}(\underline{\alpha})\right| \leqslant N$, for all $\sigma$ as we cannot align the ordering of complex and $p$-adic roots. Thus using a $p$-adic precision $k$ such that $p^{k}>2 N$ means that we can easily find (the unique) $\theta \in \mathbb{Z}$ such that $F(\underline{\alpha})=\theta \bmod p^{k}$ and $|\theta|<N$.

Proving that $F(\underline{\alpha})=\theta$ is equivalent to showing that $R_{F}(\theta)=0$. Since $R_{F} \in \mathbb{Z}[t]$ and $\theta \in \mathbb{Z}$, we have $R_{F}(\theta) \in \mathbb{Z}$. From $F(\underline{\alpha})=\theta \bmod p^{k}$ we get $p^{k} \mid R_{F}(\theta)$, while on the other hand $\left|R_{F}(\theta)\right| \leqslant$ $(|\theta|+N)^{(G: H)}$, so either $R_{F}(\theta)=0$ or

$$
p^{k} \leqslant(|\theta|+N)^{(G: H)},
$$

so we can easily compute $k$ large enough to prove $R_{F}(\theta)=0$. Unfortunately, $k=O(G: H)$, so $k$ is too large to be useful in general. Similarly to the use of short cosets (see Remark 7.1) we apply a hybrid approach. We choose $k$ large enough to find $\theta$, that is $k=O(\log N)$, and rely on a final proof step to verify the computation.

\subsection{Checking unproven steps}

In the case that $(G: H)$ is huge we have two problems when we apply Corollary 3.4. On the one hand the set $G / / H$ of coset representatives of $G / H$ is too big and on the other hand the needed $p$-adic precision depends exponentially on the index $(G: H)$, see (7.2). In our actual implementation we only consider the short cosets in Remark 7.1 and we replace the exponent in (7.2) by a small number like 10. Using these two modifications we are able to do the corresponding computations for the Stauduhar step. In order to get a mathematical proof for our computations we have two problems. Firstly, we apply Corollary 3.4, but we cannot check if the resolvent polynomial $R_{F}$ is squarefree by only considering some of its roots. Secondly, by only using exponent 10 instead of $(G: H)$ we cannot prove that $\theta$ is a rational integer. In both cases the probability that we are wrong is small, but this does not give a mathematical justification that $\operatorname{Gal}(f)$ is contained in a conjugate of $H$. In order to get this we change the method and use Theorem 3.2 for larger degree factors $A$ (and a different $H$ ).

For simplicity we assume we have a subgroup chain $G=: H_{0}>H_{1}>\ldots>H_{r}$ as a result of the algorithm with only one (the first) step being unproven. We expect that $\operatorname{Gal}(f)=H_{r}$, but strictly, at this point we only have the following two facts: $\operatorname{Gal}(f) \subseteq G=H_{0}$ and if $\operatorname{Gal}(f) \subseteq H_{1}$, then $\operatorname{Gal}(f)=H_{r}$. Typically, this is the result of $\left(H_{0}: H_{1}\right)$ being too large to verify the resolvent to be squarefree or the derived precision being too large to verify the rationality of the root. The correctness of the other steps is dependent on the correctness of the first step. The case of several unproven steps, due to several large indices, can be handled analogously.

We proceed as follows. We try to find a subgroup $U$ of $G$ such that $H_{r}$ acts intransitively on the cosets of $G / U$. Then we compute the resolvent polynomial $R_{F}$ for the group pair $U \leqslant G$ and some $G$-relative $U$-invariant $F$. If $R_{F}$ is squarefree and reducible then this already proves that $\operatorname{Gal}(f)$ is a proper subgroup of $G$. Since we expect that the Galois group is $H_{r}$ we can easily compute the elements $\sigma_{1}, \ldots, \sigma_{m} \in G$ which give the factor $A$ in Theorem 3.2. Therefore we can easily compute an approximation of the expected factor, hence, by rounding, the expected factor in $\mathbb{Z}$. Finally, we use exact trial division and then descend to a smaller subgroup of $G$ by using this theorem. When the stabilizer is $H_{i}$ for some $1 \leqslant i \leqslant r$, our computation is finished successfully, otherwise we replace $G$ by the stabilizer and restart this step. 
It might be difficult to find a good subgroup $U$. Good candidates are intransitive subgroups of $G$, see $[12, \S 5]$. In Example 3.6 we used a polynomial acting on 4 -sets. These $r$-set polynomials have the advantage that we can compute them quickly symbolically.

\subsection{Overall algorithm}

To quickly summarize the overall algorithm for irreducible integral polynomials $f$; we start be factoring $f$ modulo several primes $p$ in order to find a prime such that the least common multiple of the degrees of the factors is not too large (and not too small) and that $f$ remains squarefree, fixing such a prime to then compute approximations to the roots in the $p$-adic field as well as the permutation of the roots corresponding to the Frobenius of the $p$-adic field. Furthermore, if the Galois group is $\mathbf{S}_{n}$ or $\mathbf{A}_{n}$, this too is typically detected just from the degrees of the modulo $p$-factors. Finally, approximations to the complex roots of $f$ are obtained as well.

The next step, as outlined above, is to derive a suitable starting group for the Stauduhar iteration. Here, we compute all subfields of the stem field $E=\mathbb{Q}[x] / f$ of $f$, the corresponding block systems and the largest transitive group $G$ admitting those blocks.

The third step is the iteration of the Stauduhar test in $\S 7.2$ above. As a result, have a chain of subgroups with the properties described in $\S 7.4$ above, hence we apply those techniques to verify the result.

\subsection{Reducible polynomials}

Most of the outlined method applies to reducible polynomials as well, the key difference is that the groups occurring are naturally intransitive, which excludes most of the special invariants. Let $f=\prod f_{i}$ be squarefree and monic. We start by fixing a common splitting field $K$, and computing the roots of $f$ and the Galois groups $G_{i}=\operatorname{Gal}\left(f_{i}\right) \subset \mathbf{S}_{\alpha}$. Galois theory now states that Gal $f<\prod G_{i}$, so we restart the algorithm above with $f$ and $G:=\prod G_{i}$ as a starting group. We note that only subgroups $H<G$ that project onto the full Galois groups of the factors need to be investigated, that is, the final group is a subdirect product of the $G_{i}$.

\subsection{Other fields}

Most of the abstract theory described in this paper applies for all infinite ground fields as well, hence the algorithm carries over to different applications. However, there are a few remarks in order: the actual performance of the overall algorithm depends critically on the splitting field chosen. In the case of $p$-adic fields, this is a well-studied situation with excellent algorithms already known. In the case of Laurent-series over finite fields, occurring naturally as splitting fields for polynomials in $f \in \mathbb{F}_{q}(t)[x]$, the situation is similar. However, in more general fields, good descriptions of possible splitting fields are not quite that easily obtained. In particular, apart from efficiency considerations, the test for 'rationality of the resolvent root' needs to be adapted. Finally, it should be noted that several of the 'special invariant' listed above will not work in small characteristic (in particular in characteristic 2), hence the efficiency of the method is endangered.

\section{Numerical results}

In order to test the procedure outlined in this paper, we applied it to the complete contents of a database of polynomials [19] with known Galois groups (http://galoisdb.math.upb.de). This database contains explicit examples, sometimes many, for most groups of degree $\leqslant 23$. For more than $10^{6}$ polynomials, a total of 4835 different Galois groups have been computed. In 
this range, for 4624 groups the average runtime was less than $5 \mathrm{~s}$. Only five groups took more than $30 \mathrm{~s}$ to compute.

Let us look at an explicit example in detail. Let

$$
f:=x^{20}-308 x^{16}+33396 x^{12}-1554608 x^{8}+28579232 x^{4}-113379904
$$

with Galois group $20 T 684$ of order 61440 . We start by factoring $f$ modulo several small primes to select $p=89$ for our splitting field which is an unramified cubic extension of $\mathbb{Q}_{89}$. Next, subfields are computed, and we recurse by computing the Galois group of the degree 10 subfield first. Using the subfield data, we conclude that the Galois group of $f$ is a subgroup of $20 T 992$ of order $2^{17} \cdot 3 \cdot 5$. This group has six maximal transitive subgroups, of which only one is a candidate for the target groups, the others can be excluded by block systems or intersections with other known groups. The only group to test further is isomorphic to $20 T 807$ of index $2^{4}$; for this pair of groups we construct a special invariant using $\S 5.6$. The group $20 T 807$ now has eight maximal transitive subgroups, two of which we need to test further. For both subgroups, both isomorphic to $20 T 684$ of index 2, our algorithm fails to find special invariants, thus uses the generic ones from $\S 4$. Unfortunately, on evaluation of those invariants, we detect duplicate values, hence have to resort to Tschirnhaus transformation. In this example, we end up trying up to ten different transformations of degree up to 7 before we find one to remove the duplicate values, hence makes the resultant squarefree and a descent is found. The resulting group again has four maximal transitive subgroups, none of which however are possible, thus the computation terminates. The 'long' runtime here is a result of the generic invariants on the one hand and the need for Tschirnhaus transformations on the other. By construction, the generic invariants chosen are of minimal degree but need $>500000$ multiplications for a single evaluation. Due partly to the Tschirnhaus transformations, a $p$-adic precision of $>60$ digits is used which then explains the runtime.

Comparing this to other polynomials with the same group, we see that the runtime varies substantially $(20-240 \mathrm{~s})$ which is due to the number of Tschirnhaus transformations used: this depends on the polynomials and not (directly) on the group. In this example, the 'nice' structure of the polynomial with lots of zero coefficients indirectly causes the transformations, while we could 'easily' fix this by a transformation of the original polynomial, this would also incur a drastic growth of the coefficients, thus rendering this mostly useless.

Overall, the runtime can be seen to depend mainly on the groups as this determines the invariants and the descent tree transversed. Long runtimes typically are the result of bad invariants (generic invariants, frequently if the groups are very similar, that is small index). Large index subgroups, while posing a potential problem for the verification, are frequently easy to compute with: the short cosets reduce the number of candidates dramatically and the vastly differing groups make finding of invariants easy.

\section{Future work}

The algorithm, as presented here, has two major weaknesses: it needs to find 'good' invariants and it 'needs' a small index in order to have verifiable results. Thus more work is needed to increase the number of 'special' invariants. In fact, work in this direction has already commenced, for example Elsenhans [10] found better invariants for pairs of intransitive groups and for certain (large) pairs of 2-groups. In order to address the verification problem, maybe the use of non-linear factors of the resolvent polynomials as demonstrated in Example 3.5 should be investigated further.

However, as of now, we have a degree independent complete algorithm to compute Galois groups of univariate polynomials. The algorithm is very efficient and has been used on polynomials of degree $>100$ already. 


\section{References}

1. I. Abdeljaouad, 'Calculs d'invariants primitifs de groupes finis', Theor. Inform. Appl. 33 (1999) 59-77.

2. M. Bridson and C. Miller, 'Structure and finiteness properties of subdirect products of groups', Proc. Lond. Math. Soc. (3) 90 (2009) 631-651.

3. D. CASPERSON and J. MCKay, 'Symmetric functions, m-sets, and Galois groups', Math. Comput. 63 (1994) 749-757.

4. H. Cohen, A course in computational algebraic number theory (Springer, Berlin, 1993).

5. J. H. Conway, A. Hulpke and J. MCKay, 'On transitive permutation groups', LMS J. Comput. Math. 1 (1998) 1-8.

6. H. Derksen and G. Kemper, 'Computational invariant theory', Encyclopaedia of mathematical sciences. Invariant theory and algebraic transformation groups (Springer, Berlin, 2002).

7. J. Dixon and B. Mortimer, Permutation groups (Springer, Berlin-Heidelberg-New York, 1996).

8. T. Dokchitser and V. Dokchitser, 'Identifying Frobenius elements in Galois groups', Algebra Number Theory $7 / 6$ (2013) 1325-1352.

9. Y. Eichenlaub, 'Problèmes effectifs de théorie de Galois en degrés 8 à 11', Thèse, Université Bordeaux 1, 1996.

10. A.-S. Elsenhans, 'Invariants for the computation of intransitive and transitive Galois groups', J. Symbolic. Comput. 47 (2012) 315-326.

11. K. GeißLER, 'Berechnung von Galoisgruppen über Zahl- und Funktionenkörpern', PhD Thesis, Technische Universität Berlin, 2003.

12. K. Geißler and J. Klüners, 'Galois group computation for rational polynomials', J. Symbolic. Comput. 30 (2000) 653-674.

13. K. Girstmair, 'On the computation of resolvents and Galois groups', Manuscripta Math. 43 (1983) 289-307.

14. K. Girstmair, 'On invariant polynomials and their application in field theory', Math. Comput. 48 (1987) $781-797$.

15. M. van HoeiJ, J. Klüners and A. Novocin, 'Generating subfields', J. Symbolic. Comput. 52 (2013) $17-34$.

16. G. Kemper and A. Steel, 'Some algorithms in invariant theory of finite groups', Computational Methods for Representations of Groups and Algebras, Euroconference in Essen, April 1-5 1997, Progress in Mathematics 173 (eds P. Dräxler, G. O. Michler and C. M. Ringel; Birkhäuser, 1997).

17. J. KLÜNERS, 'Über die Berechnung von Automorphismen und Teilkörpern algebraischer Zahlkörper', PhD TU-Berlin (1997).

18. J. Klüners and G. MALle, 'Explicit Galois realization of transitive groups of degree up to 15', J. Symbolic. Comput. 30 (2000) 675-716.

19. J. Klüners and G. Malle, 'A database for field extensions of the rationals', LMS J. Comput. Math. 4 (2001) 182-196.

20. R. Loos, 'Computing in algebraic extensions', Computer algebra. Symbolic and algebraic computation, Computing Supplementum 4 (eds B. Buchberger, G. E. Collins and R. Loos; Springer, Wien, 1982) 173-187.

21. B. Schmalz, 'Verwendung von Untergruppenleitern zur Bestimmung von Doppelnebenklassen', Bayreuther Math. Schriften 31 (1990) 109-143.

22. L. Soicher, 'The computation of Galois groups', M. Comp. Sci. Thesis, Concordia University, Montreal, 1981. http://www.maths.qmul.ac.uk/ leonard/mcompsc_soicher.pdf.

23. R. Stauduhar, 'The determination of Galois groups', Math. Comput. 27 (1973) 981-996.

24. K. Yokoyama and G. Renault, 'A modular method for computing the splitting field of a polynomial', ANTS 2006, Lecture Notes in Computer Science 4076 (Springer, 2006) 124-140.

\section{Claus Fieker}

Faculty of Mathematics

University of Kaiserlautern

P.O. Box 3049

D-67653 Kaiserslautern

Germany

fieker@mathematik.uni-kl.de
Jürgen Klüners

Mathematisches Institut der

Universität Paderborn

Warburger Str. 100

D-33098 Paderborn

Germany

klueners@math.uni-paderborn.de 